\title{
Spectroscopic diagnostics of superthermal electrons with high-number harmonic EC radiation in tokamak reactor plasmas
}

\author{
P.V. Minashin ${ }^{1, \mathrm{a}}$, A.B. Kukushkin ${ }^{1,2}$ \\ ${ }^{1}$ Tokamak Physics Institute, NRC Kurchatov Institute, Moscow, Russia \\ ${ }^{2}$ National Research Nuclear University MEPhI, Moscow, Russia
}

\begin{abstract}
A method of spectroscopic diagnostics of the average perpendicular-to-magnetic-field momentum of the superthermal component of the electron velocity distribution (EVD), based on the high-numberharmonic electron cyclotron (EC) radiation, is suggested for nuclear fusion-reactor plasmas under condition of a strong auxiliary heating (e.g. in tokamak DEMO, a next step after tokamak ITER). The method is based on solving an inverse problem for reconstruction of the EVD in parallel and perpendicular-to-magnetic-field components of electron momentum at high and moderate energies responsible for the emission of the highnumber-harmonic EC radiation.
\end{abstract}

\section{Introduction}

Spectroscopic diagnostics of electron temperature from the low EC harmonics of thermal EC radiation is widely used in the facilities for magnetic confinement of hightemperature plasmas (tokamaks, stellarators). In tokamaks, the diagnostics is based on the (approximate) one-to-one correspondence between three quantities: toroidal major radial coordinate, toroidal magnetic field, and the EC frequency of registered radiation [1], [2]. For low-number harmonics of fundamental cyclotron frequency (usually, the $1^{\text {st }}$ and $2^{\text {nd }}$ ones) the emitted EC radiation is strongly trapped in a rather thin layer of plasma on the line-of-sight (usually, perpendicularly to the toroidal magnetic field) of the detector. For thermodynamic equilibrium (Maxwellian velocity distribution for electrons) one has similar equilibrium (Planck distribution) for radiation intensity which - for the smallness of wave quantum energy as compared to temperature (that is definitely valid for EC radiation) reduces to Rayleigh-Jeans direct proportionality between intensity and temperature. Thus, the frequency profile of the registered thermal radiation at a certain low-number harmonic appears to give the line-of-sight profile of electron temperature. The deviations of electron velocity distribution from a Maxwellian distort this correspondence and may be a source of sometimes observed difference of temperature values inferred from the local spectrum of the laser radiation Thomson scattering and the EC radiation intensity at the respective point of plasma. However, the non-Maxwellian effects are relatively small for the frequency domain where thermal EC radiation dominates.
The strong effects of a non-Maxwellian EVD may be observed at the relativistically down-shifted frequencies (including those lower than the $1^{\text {st }}$ and $2^{\text {nd }}$ harmonic for the low-magnetic-field side of tokamak plasma) where the EC radiation is emitted by the fast, non-thermal electrons at the periphery and may escape from plasma. An example of treating these effects for the reconstruction of an essentially non-Maxwellian EVD may be found in [3], [4] for interpretation of the data from tokamak T-10. However, the use of this effect for diagnostics of the edge plasma in fusion reactors is possible only in regimes with low plasma density at the plasma edge.

The studies of non-Maxwellian effects at EC harmonics $n>2$ have a long history, see, e.g., [5]. The complexity of this case stems from several issues: the transient regime of radiation transfer, namely, from optical thickness (strong trapping of ECR) at EC harmonics $n=1$ and $n=2$ to optical transparency at highnumber harmonics; a decrease of the spectral gap between, and overlapping of, the neighboring harmonics of the thermal EC radiation. In tokamak-reactors one has a simplification in the sense that for $\mathrm{n}>3$ the EC radiation has the following features: (a) the spectrum of the outgoing EC radiation is a smooth function, due to a strong overlapping of harmonics in a high-temperature fusion plasma and (b) the radiative transfer at the frequencies which are most important for EC radiation losses, takes place in the regime of a low optical thickness and strong reflections from the first wall [6][9]. The latter, however, makes the radiative transport essentially nonlocal, and the identification of nonMaxwellian effects is feasible only for central plasma,

\footnotetext{
$\overline{{ }^{a} \text { Corresponding author Minashin_PV}}[$ at]nrcki.ru
} 
with the highest temperature, because only this radiation has a peculiar spectral range (namely, high-number harmonics) in the spectrum of outgoing radiation.

In contrast to the present day tokamaks, the $\mathrm{EC}$ wave emission by the plasma can significantly contribute to the local electron power balance in central part of plasma column for high temperatures expected in DEMO and steady-state regimes of ITER operation (see, e.g., [10], [11]). When central temperature increases to $\mathrm{T}_{\mathrm{e}}(0) \sim 30 \mathrm{keV}$ the local EC power loss becomes a substantial part of heating from fusion alpha-particles and is close to the total auxiliary heating. Also, the fast increase of the EC losses with increasing temperature may have a positive impact on stabilization of fusion burning [12], keeping fusion power and divertor loads at moderate level for all imaginary temperature excursions, that is important for long pulse operation scenarios [13]. These conclusions have been made in the frame of modeling with the neglect of deviations of the EVD from a Maxwellian. The impact of possible deviations of the EVD from a Maxwellian on the EC power losses has been analyzed in [14]-[16]. First self-consistent calculation of the plasma-produced EC radiation and superthermal electron kinetics was carried out in [17]. The problem of self-consistent modeling of electron kinetics, EC wave propagation and EC power losses in tokamaks is discussed in [18] (see also references therein). This assumes using the Fokker-Planck codes for numerical modeling of electron kinetics (e.g. the CQL3D code [19]) with allowance for both the injected EC wave at low-number harmonics and the plasma-produced EC radiation which in tokamak-reactors is dominant at moderate- and high-number EC harmonics. An example of using the CQL3D for interpretation of diagnostic data (non-Maxwellian effects in the Thomson scattering in tokamak TCV under ECRH and ECCD conditions) may be found in [20]. In general, one has to combine various diagnostic data to reconstruct the deviations of the EVD from a Maxwellian.

In the present paper, we suggest a new method of spectroscopic diagnostics of the average perpendicularto-magnetic-field momentum of the superthermal component of the EVD, based on the high-number harmonic EC radiation from nuclear fusion-reactor plasmas under condition of a strong auxiliary heating (e.g. in tokamak DEMO, a next step after tokamak ITER). The method is based on solving an inverse problem for reconstruction of the EVD in parallel and perpendicular-to-magnetic-field components of electron momentum at high and moderate energies responsible for the emission of the high-number-harmonic EC radiation.

\section{Diagnostics of electron temperature from EC radiation spectrum for Maxwellian plasmas}

We start our consideration with an analysis of the possibility to diagnose the electron temperature of the central hot plasma in a fusion reactor from the EC radiation spectrum for purely Maxwellian plasmas. In this case it is possible to observe the impact of central temperature on the EC spectrum in a certain range of frequencies. One can expect the dependence of spectrum's slope with increasing frequency on the absolute value of the central temperature. We illustrate this effect on the example of a variation of the electron temperature profile around that obtained in [21] for selfconsistent modeling of plasma parameters in a DEMOtype tokamak, with the help of the CRONOS suit of codes [22] for plasma equilibrium and transport modeling. The results of such an analysis are shown in figures 1-4.

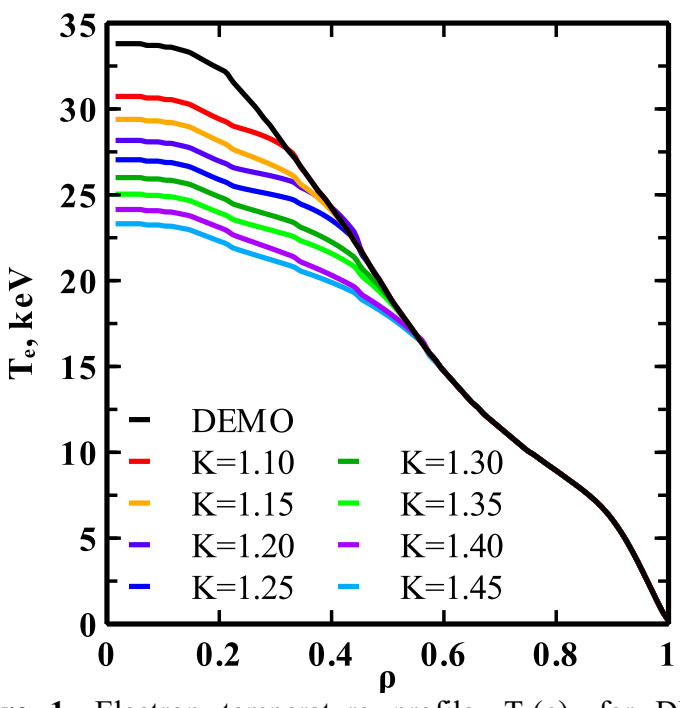

Figure 1. Electron temperature profile, $T_{e}(\rho)$, for DEMO scenario 2 with EC heating and EC current drive [21] (black line) predicted by the CRONOS code, and a set of hypothetical temperature profiles obtained from the above profile by a proportional decrease in the range $\rho \leq 0.5, \mathrm{~T}_{\mathrm{e}}(\rho \leq 0.5) / \mathrm{K}$, using a spline interpolation with the original profile for $\rho>0.5$. Parameter $\rho$ is a square root of the normalized toroidal magnetic flux. Global characteristics of DEMO operation scenarios [21]: major radius $\mathrm{R}_{0}=7.5 \mathrm{~m}$, minor radius $\mathrm{a}=2.5 \mathrm{~m}$, elongation $\mathrm{k}_{\text {elong }}=1.9$, triangularity $\delta=0.47$, vacuum toroidal magnetic field on the toroidal axis of vacuum vessel $\mathrm{B}_{0}=6 \mathrm{~T}$, plasma current $\mathrm{I}_{\mathrm{p}}=19 \mathrm{MA}$, electron density $\mathrm{n}_{\mathrm{e}}(0)=1.3 \quad 10^{20} \mathrm{~m}^{-3}$, $\left.<\mathrm{n}_{\mathrm{e}}>=1.010^{20} \mathrm{~m}^{-3}\right)$.

To calculate the spectrum of the outgoing EC radiation we use the CYNEQ code [7], [23], [24] while the EXACTEC code [8] was used in the modeling [21]. Note that comparison of the codes for calculation the power losses profiles was carried out in [25] and [26] for various representation of the effective magnetic field which is relevant to the existing practice of the 1D transport modeling of tokamak plasma (e.g., ASTRA [27] and CRONOS [22]). This field, $\mathrm{B}(\rho)$, is obtained from the $2 \mathrm{D}$ magnetic field, $\mathrm{B}(\rho, \theta)$, as a function of the normalized toroidal flux through the magnetic surface, $\rho$, and poloidal angle, $\theta$, by averaging over magnetic flux surface. In [25] the benchmarking of the codes was done for $\mathrm{B}(\rho)=$ const and given profiles of density and temperature, whereas in [26] a comparison is made for the results obtained with account of the effects of 2D magnetic field and plasma equilibrium calculated selfconsistently with plasma transport, including the EC power losses.

In the CYNEQ model [7], which modifies and improves the nonlocal EC radiation transport model [28], 
the intensity of the outgoing radiation is described by the following formula (here for simplicity the mixing of the EC wave modes, caused by wave reflection, is neglected):

$$
\begin{gathered}
I_{\text {esc }}(\omega, \zeta)=\frac{\left\langle\int \frac{d \Omega_{n}}{4 \pi} q_{\varsigma}(\mathbf{r}, \Phi)\right\rangle_{V_{\text {esc }}}}{\int \frac{d \Omega_{n}}{4 \pi} \int \frac{\mathbf{n} \cdot d \mathbf{S}_{w}}{V_{\text {esc }}}\left(1-R_{w}\right)+\left\langle\int \frac{d \Omega_{n}}{4 \pi} \kappa_{\varsigma}(r, \Phi)\right\rangle_{V_{\text {esc }}}}, \\
I_{\text {esc }}(\omega)=\sum_{\varsigma=X, O} I(\omega, \varsigma),
\end{gathered}
$$

where $\Phi=\{\omega, \mathbf{n}, \varsigma\}, \omega$ and $\mathbf{n}=\mathbf{k} / \mathrm{k}$ - wave frequency and direction of propagation, $\mathbf{k}$ - wave vector, index $\varsigma=\mathrm{X}, \mathrm{O}$ denotes, respectively, the extraordinary $(\mathrm{X})$ and ordinary $(\mathrm{O})$ wave types, $\kappa(\mathbf{r}, \phi)$ is the EC radiation absorption coefficient, $\mathrm{q}(\mathbf{r}, \phi)$ is the power density of the EC radiation source, $V_{\text {esc }}$ is a part of the plasma volume where the EC radiation can propagate almost without absorption, with taking into account the wave cut-off, $<>_{\text {Vesc }}$ is an averaging over volume $V_{\text {esc }}, S_{w}$ - area of vacuum chamber inner surface (the surface integral in the denominator of the fraction (1) is taken over the semisphere where $\left.\left(\mathbf{n} \cdot \mathrm{d} \mathbf{S}_{\mathrm{w}}\right)>0\right), \mathrm{R}_{\mathrm{w}}$ is reflection coefficient of the EC radiation from the wall.

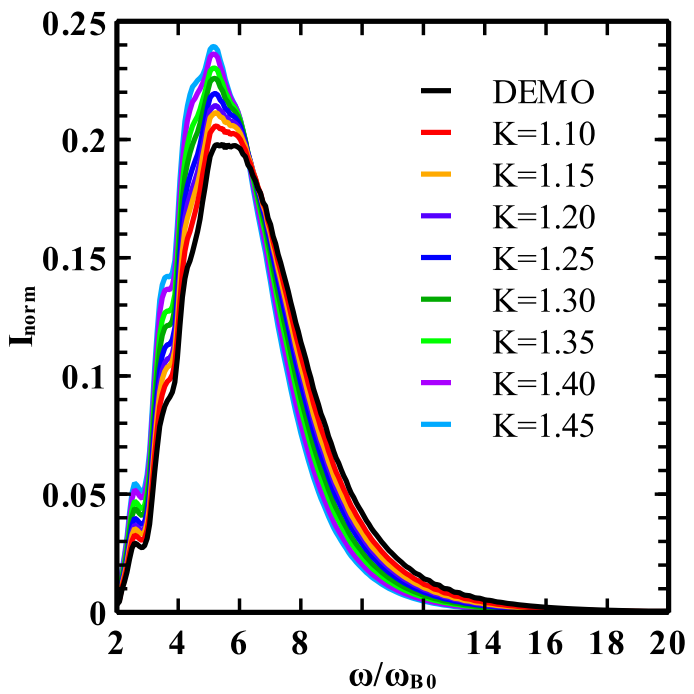

Figure 2. Normalized intensity of the outgoing EC radiation, $I_{\text {norm }}$, as a function of normalized frequency, $\omega / \omega_{\mathrm{B} 0}\left(\omega_{\mathrm{B} 0}\right.$ is the fundamental $\mathrm{EC}$ frequency for magnetic field $\mathrm{B}_{0}$ ), calculated by the CYNEQ code [7], [23], [24] for DEMO scenario 2 [21], $\mathrm{R}_{\mathrm{w}}=0.7$ (black line) and for the same parameters and the modified $\mathrm{T}_{\mathrm{e}}$ profiles (colored lines) (see Fig. 1).

It is seen from Figure 2 that there is a possibility to distinguish between various central temperatures in the range of frequency $5 \leq \omega / \omega_{\mathrm{B} 0} \leq 8$ : here the slope of the spectrum shows a regular monotonic behaviour. One can compare the respective values and restore the central temperature. Despite the slope (i.e. derivative) has substantial local fluctuations, the averaged values of the slope exhibit regular behaviour (Figs. 3 and 4).

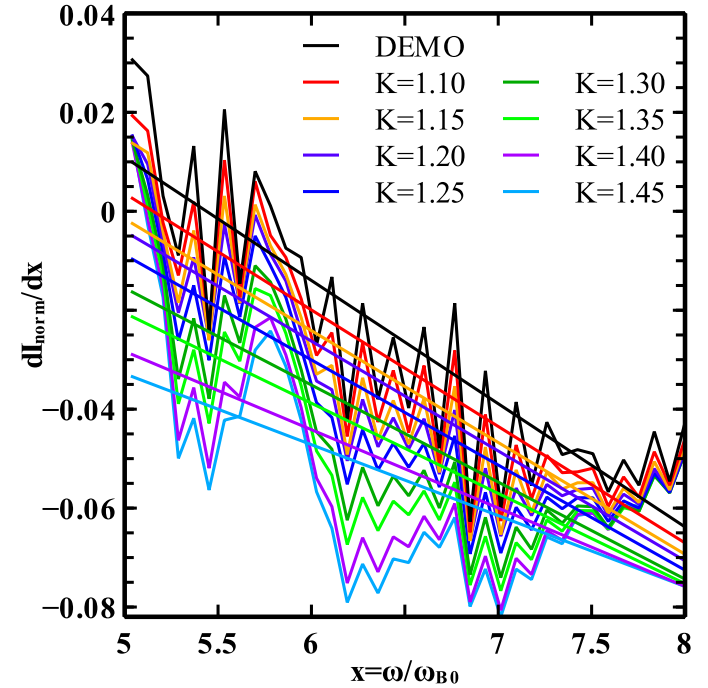

Figure 3. Derivative of the normalized intensity of the outgoing EC radiation from Fig. 2 with respect to its argument $x=\omega / \omega_{B 0}$ (solid lines). The notations are the same as in Fig. 2. Also the linear least-square fitting of the curves is shown (dotted lines).

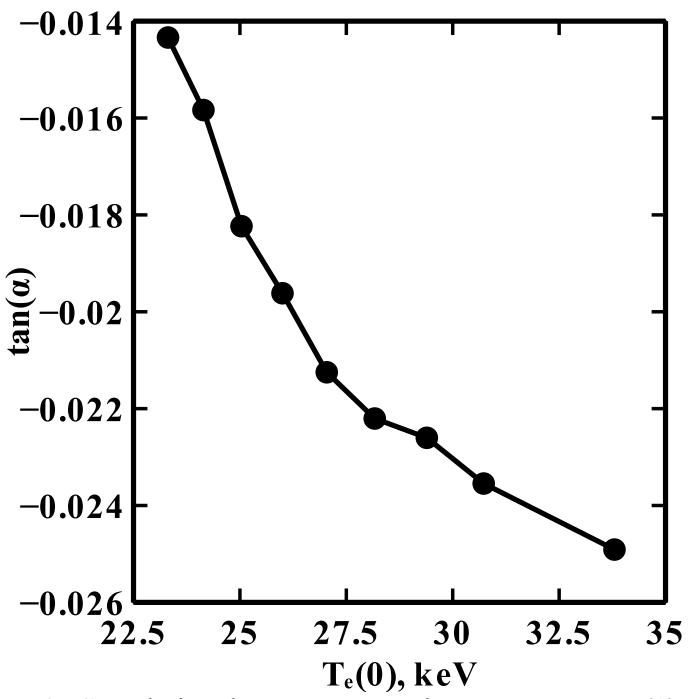

Figure 4. Correlation between central temperature, $\mathrm{T}_{\mathrm{e}}(0)$, and $\tan (\alpha)$, the slope of the linear approximation of the derivative of the normalized intensity of the outgoing EC radiation in Fig. 3 in the range $5 \leq \omega / \omega_{\mathrm{B} 0} \leq 8$. The Pearson's linear correlation coefficient is equal to -0.94 .

\section{Diagnostics of electron velocity distribution parameters from EC radiation spectrum in fusion-reactor non-Maxwellian plasmas}

The optimistic conclusions of the previous section are based on the use of the known functional form of the EVD. In the case of arbitrary EVD the capability to reconstruct the main parameters of the EVD are restricted to those parameters, which are most sensitive to EC radiation, first of all, to average perpendicular-tomagnetic-field momentum of non-thermal electrons.

Numerical modeling of the EC radiation in the presence of an anisotropic distribution of superthermal electrons is carried out here with the modified CYNEQ code [7], [23], [24]. We assume the electron distribution 
function to be a sum of a Maxwellian distribution function for the bulk plasma and a bi-Maxwellian anisotropic distribution of superthermal electrons:

$$
\begin{gathered}
\mathrm{f}(\rho, \mathbf{p})=\left(1-\delta_{\mathrm{Hot}}(\rho)\right) \cdot \mathrm{f}_{\text {Maxw }}+\delta_{\text {Hot }}(\rho) \cdot \mathrm{f}_{\mathrm{Hot}}\left(\rho, \mathrm{p}_{\|}, \mathrm{p}_{\text {। }}\right), \\
\mathrm{f}_{\mathrm{Hot}}\left(\rho, \mathrm{p}_{\|}, \mathrm{p}_{\perp}\right)=\text { Const } \cdot \exp \left[-\mathrm{m}_{\mathrm{e}} \mathrm{c}^{2}\left(\frac{\sqrt{1+\mathrm{p}^{2}}-1}{\mathrm{p}^{2}}\right) .\right. \\
\left.\cdot\left(\frac{\mathrm{p}_{\|}^{2}}{\mathrm{~T}_{\|}(\rho)}+\frac{\mathrm{p}_{\perp}^{2}}{\mathrm{~T}_{\perp}(\rho)}\right)\right], \\
\int \mathrm{f}(\rho, \mathbf{p}) \mathrm{d} \mathbf{p}=\mathrm{n}_{\mathrm{e}}(\rho),
\end{gathered}
$$

where $\mathbf{p}$ and $\varepsilon$ is the momentum and the energy of an electron; the signs $\|, \perp$ denote parallel and perpendicular-to-magnetic-field components of a vector, respectively; $\delta_{\mathrm{Hot}}(\rho)$ and $\mathrm{T}_{\|, \perp}(\rho)$ profiles are as follows:

$$
\begin{gathered}
\mathrm{F}(\rho)=(\mathrm{F})_{\max } \operatorname{EXP}\left[-\left(\rho-\rho_{0}\right)^{2} /(\Delta \rho)^{2}\right], \\
\mathrm{F}=\left\{\mathrm{T}_{\perp, \|}, \delta_{\mathrm{Hot}}\right\}, \\
\rho_{0}=0, \Delta \rho=0.3, \\
\left(\delta_{\mathrm{Hot}}\right)_{\max }=0.1, \\
\left(\mathrm{~T}_{\|}\right)_{\max }=90 \mathrm{keV},\left(\mathrm{T}_{\perp}\right)_{\max }=60 \mathrm{keV} .
\end{gathered}
$$

The respective intensity of the outgoing EC radiation exhibits the presence of a "tail" of the spectrum at highnumber harmonics (Fig. 5).

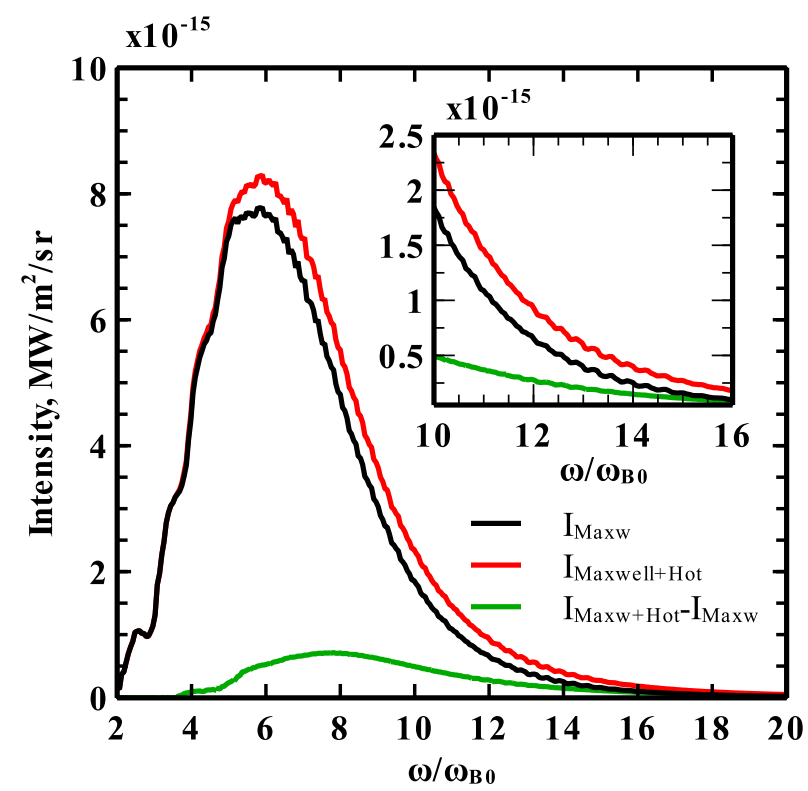

Figure 5. Intensity of the outgoing $\mathrm{EC}$ radiation, as a function of normalized frequency, $\omega / \omega_{\mathrm{B} 0}$, calculated by the CYNEQ code [7], [23], [24] for DEMO scenario 2 in Ref. [21] for the Maxwellian electron distribution function (black line) and distribution function of eqs. (3)-(7) with the same parameters of the bulk Maxwellian and a fraction of superthermal electrons (red line). In addition, the difference between two spectra is shown (green dashed line). Inset shows in more detail the range of frequencies which are of interest to the proposed spectroscopic diagnostics.

The proposed spectroscopic diagnostics is based on the solution of an inverse problem for reconstruction of the EVD in parallel and perpendicular-to-magnetic-field components of electron momentum at high and moderate energies responsible for the emission of the high-number- harmonic EC radiation (similarly to the inverse problem solved in [3]). In such a formulation we assume that

- parameters of the EVD of the bulk Maxwellian plasma are known from other diagnostics, presumably, Thomson scattering, and

- we can determine parameters of the EVD only at high enough energy of electrons and actually only in the central plasma.

In the frame of the CYNEQ model the inverse problem is formulated as follows. For the high-number harmonics the intensity of the outgoing EC radiation, described by eqs. (1)-(7), may be simplified, due to a small absorption at these frequencies, and takes the form of a linear dependence of the measured intensity on the EVD of superthermal electrons:

$$
\begin{gathered}
\mathrm{I}_{\text {esc }}(\omega)=\mathrm{I}_{\text {esc }}^{\mathrm{Maxw}}(\omega)+\mathrm{I}_{\text {esc }}^{\mathrm{Hot}}(\omega), \\
\mathrm{I}_{\mathrm{esc}}^{\mathrm{Hot}}(\omega)=\sum_{\varsigma=\mathrm{X}, \mathrm{O}} \frac{\int \frac{\mathrm{d} \Omega_{\mathrm{n}}}{4 \pi} \mathrm{dV} \mathrm{dp} \mathrm{q}_{\varsigma}^{\mathrm{Hot}}(\mathbf{r}, \Phi)}{\frac{\mathrm{d} \Omega_{\mathrm{n}}}{4 \pi} \int \mathbf{n} \cdot \mathrm{d} \mathbf{S}_{\mathrm{w}}\left(1-\mathrm{R}_{\mathrm{w}}(\omega, \mathbf{n})\right)} \\
\left\langle\frac{\mathrm{d} \Omega_{\mathrm{n}}}{4 \pi} \mathrm{q}_{\varsigma}^{\mathrm{Hot}}\right\rangle_{\mathrm{V}_{\mathrm{esc}}}=\int \frac{\mathrm{dV}}{\mathrm{V}} \mathrm{d} \mathbf{p}\langle\eta(\mathbf{r}, \mathbf{p}, \mathbf{n}, \omega, \zeta)\rangle_{\Omega_{\mathrm{n}}} . \\
\cdot \delta_{\mathrm{Hot}}(\rho) \cdot \mathrm{f}_{\mathrm{Hot}}\left(\rho, \mathrm{p}_{\|}, \mathrm{p}_{\perp}\right),
\end{gathered}
$$

where $\mathrm{I}_{\mathrm{esc}}(\omega)$ is the intensity of the outgoing EC radiation, $\mathrm{I}_{\mathrm{esc}}^{\mathrm{Maxw}}(\omega)$ is the intensity for the Maxwellian EVD, $\eta(\mathbf{r}, \mathbf{p}, \mathbf{n}, \omega, \zeta)$ is the emissivity for a single electron, calculated using the Schott-Trubnikov formula (cf. [1]). Note that for high harmonics of the EC fundamental frequency, $\mathrm{V}_{\mathrm{esc}}$ in eq. (1) is equal to the total plasma volume, $\mathrm{V}$. The direct problem - the calculation of the EC radiation spectra for a given EVD - is the calculation of eqs. (8)-(10) for known parameters of the bulk plasma and the superthermal electrons. The inverse problem assumes determination of the superthermal electron distribution function $\delta_{\mathrm{Hot}}(\rho) \mathrm{f}_{\mathrm{Hot}}(\rho, \mathbf{p})$, for which the calculated spectrum $\mathrm{I}_{\mathrm{esc}}^{\mathrm{Hot}}(\omega)$ is the difference of the observed intensity $\mathrm{I}_{\mathrm{esc}}(\omega)$ and the calculated intensity $\mathrm{I}_{\mathrm{esc}}^{\mathrm{Maxw}}(\omega)$ for the bulk plasma. Equations (8)-(10) can be rewritten in the following operator form:

$$
\begin{gathered}
\operatorname{Mf}(\mathbf{r}, \mathbf{p})=\mathrm{I}(\omega), \\
\mathrm{I}(\omega)=\mathrm{I}_{\mathrm{esc}}(\omega)-\mathrm{I}_{\mathrm{esc}}^{\mathrm{Maxw}}(\omega), \\
\left.\sum_{\mathrm{s}=\mathrm{X}, \mathrm{O}} \frac{\int \mathrm{dV} \mathrm{d} \mathbf{p}\langle\eta\rangle_{\Omega_{\mathrm{n}}} \mathrm{f}(\mathbf{r}, \mathbf{p})}{\int \frac{\mathrm{d} \Omega_{\mathrm{n}}}{4 \pi} \int \mathbf{n} \cdot \mathrm{d} \mathbf{S}_{\mathrm{w}}\left(1-\mathrm{R}_{\mathrm{w}}\right)}\right),
\end{gathered}
$$

where linear operator $\mathrm{M}$ is acting between the space $\mathrm{R}_{\mathrm{f}}=\left\{\rho, \mathrm{p}_{\|}, \mathrm{p}_{\perp}\right\}$, in which the EVD function is set ( $f \in R_{f}$ ), and the space $Z=\{\omega\}$, in which the observed intensity is set $\left(I_{\text {esc }}(\omega) \in Z\right)$. Because of the impossibility to invert the $\mathrm{M}$ operator (there is no unique solution) we solve this inverse problem using the optimization methods. We define the objective function that measures how good the calculated spectrum fits the observed data:

$$
\phi(\mathrm{f})=\|\mathrm{Mf}-\mathrm{I}\|_{\mathrm{Z}}^{2},
$$


which represents the L-2 norm, in Z-space, of the misfit between the predicted data and the observed data. The optimization problem with the object function (14) and the nonnegative constraint is formulated as follows (nonnegative least squares):

$$
\min _{\mathrm{f}}\|\mathrm{Mf}-\mathrm{I}\|_{Z}^{2}, \mathrm{f} \geq 0
$$

The inverse problem (15) is an ill-posed one. The standard way to treat such ill-posed problems is to use the numerical regularization theory.

Here we show that if we incorporate additional information about the physics of EC radiation at high harmonics in the problem (15) we can restore some parameters of the superthermal electrons. We shall consider the simplest case of $0 \mathrm{D}$ magnetic field in the 1D radiation transport model (namely, the magnetic-surfaceaverage magnetic field is taken $\left.\mathrm{B}(\rho)=\mathrm{B}_{0}\right)$ and concentric circle magnetic surfaces, so that in eq. (13) the function $\langle\eta(\rho, \mathbf{p}, \mathbf{n}, \omega, \zeta)\rangle_{\Omega_{\mathrm{n}}}$ does not depend on the magnetic surface coordinate $\rho$. For this case the problem (8)-(10) can be solved in two steps:

- restoring the $\mathrm{q}_{=}=\mathrm{q}_{\varsigma}^{\text {Hot }}(\rho, \omega)$ function from the observed spectra $\mathrm{I}(\omega)$ via solving the inverse problem in the $\rho$-space,

- restoring the EVD function of superthermal electrons from $q=q_{\varsigma}^{\text {Hot }}(\rho, \omega)$ via solving the inverse problem in the $\left(\mathrm{p}_{\|}, \mathrm{p}_{\mid}\right)$-space.

Assuming that the most significant contribution to the $\mathrm{I}_{\text {esc }}^{\text {Hot }}(\omega)$ spectra comes from superthermal electrons located near the $\rho=0$ surface, we can start directly with the 2-nd task.

We illustrate the capability of the above algorithm on the example of the bulk plasma taken again from [21], and a superthermal electrons with parameters (4), (6)-(7). The results of such an analysis are shown in Figures 6-9 where for simplicity we consider the X-mode of EC radiation which dominates in the observed signal.

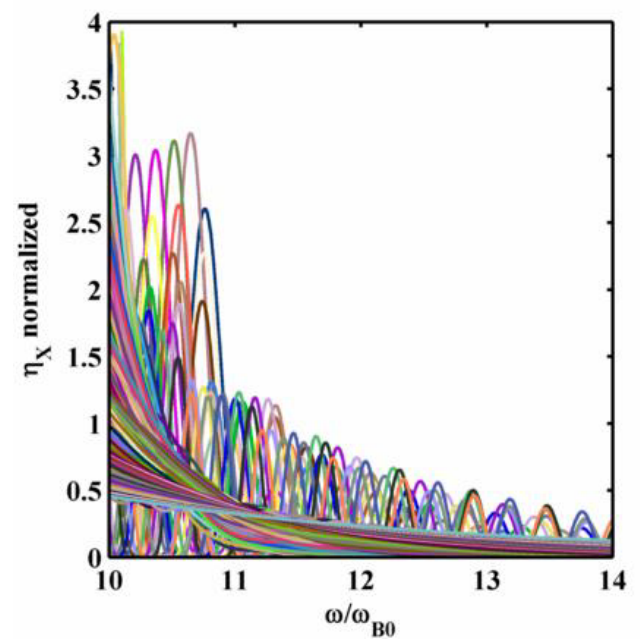

Figure 6. Emissivity for the $\mathrm{X}$-mode of $\mathrm{EC}$ radiation from a single electron, in the units of $\mathrm{e}^{2} \omega^{2} /\left(2 \pi \omega_{\mathrm{B} 0} \mathrm{c}\right)(\mathrm{c}-$ speed of light), averaged over the photon angles, as a function of normalized frequency for different cells in the $\left(\mathrm{p}_{\|}, \mathrm{p}_{\perp}\right)$ space.

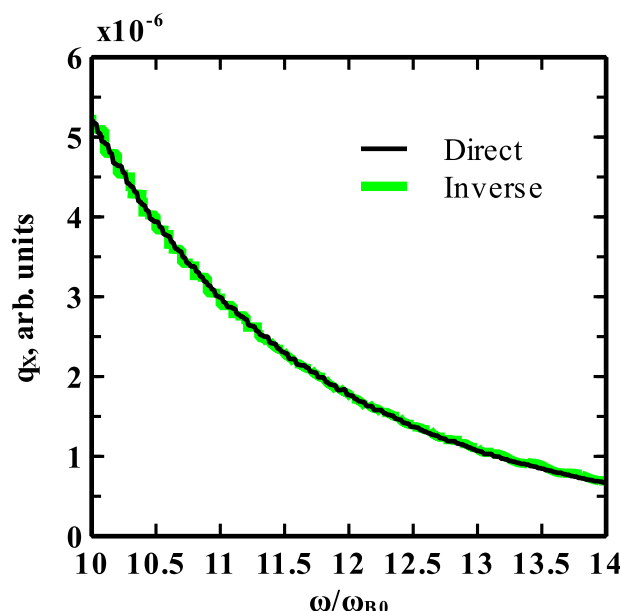

Figure 7. The power density of the spontaneous EC radiation source for the X-mode (in the units of $\mathrm{e}^{2} \omega^{2} /\left(2 \pi \omega_{\mathrm{B} 0} \mathrm{c}\right)$ ): direct calculation by the CYNEQ code for superthermal EVD (4), (6), (7), which is used as a synthetic input (i.e. "phantom" experimental) data for the proposed diagnostics (black line) and the results of solving the inverse problem (15) (green dashed line).

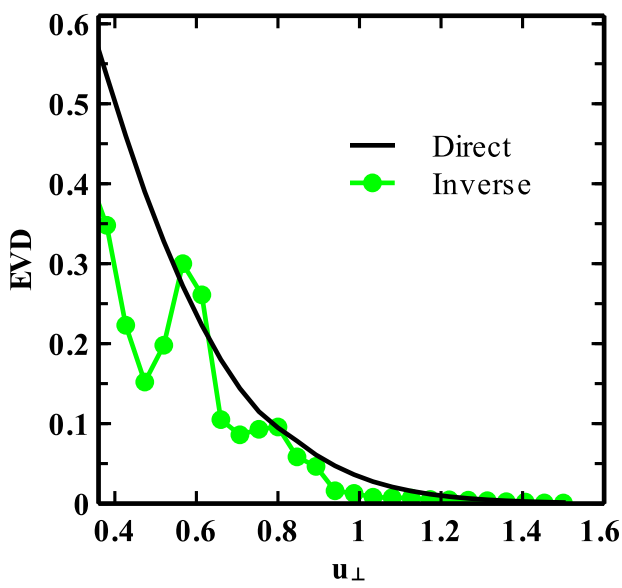

Figure 8. The EVD as a function of perpendicular-to-magneticfield component of the momentum $\mathbf{u}=\mathbf{p} / \mathrm{mc}$ for fixed values $\mathrm{u}_{\|}=0.1$ and $\rho=0$ : direct (assumed) data (4), (6), (7) (black line) and the result of solving the inverse problem (15) (green dashed line).

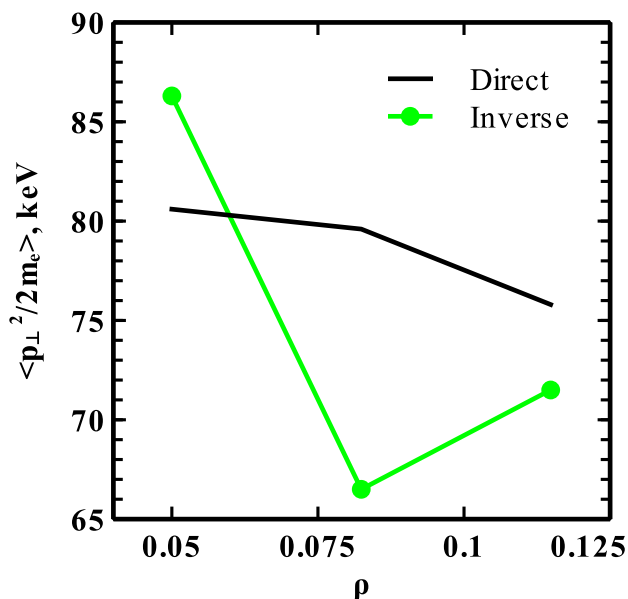

Figure 9. Averaged value of perpendicular kinetic energy as a function of the magnetic surface label $\rho$ : direct calculation for EVD (4), (6), (7) (black line) and the results of solving the inverse problem (15) (green dashed line). 


\section{Conclusions}

An analysis of the high-number-harmonic EC radiation from a hot fusion-reactor plasmas, expected under condition of a strong auxiliary heating (e.g. in tokamak DEMO, a next step after tokamak ITER), enabled us to suggest a new method of spectroscopic diagnostics of the average perpendicular-to-magnetic-field momentum of the superthermal component of the electron velocity distribution (EVD). The method is based on solving an inverse problem for reconstruction of the EVD in parallel and perpendicular-to-magnetic-field components of electron momentum at high and moderate energies responsible for the emission of the high-numberharmonic EC radiation. The well-known difficulty of solving such a problem in the case of arbitrary EVD (complexity of spectrum, radiative transfer effects for low-number harmonics and nonlocality of transport for high-number harmonics, etc.) restricted the capability to reconstruct the main parameters of the EVD to those parameters which are most sensitive to the EC radiation, namely, the average perpendicular-to-magnetic-field momentum of superthermal electrons. However, a combination of such a diagnostic with those which can determine the parameters of the bulk Maxwellian plasma (like Thomson scattering diagnostics) may provide a powerful tool for spectroscopic plasma diagnostics of fusion-reactor plasmas.

Acknowledgements. The authors are grateful to E.E. Mukhin (Ioffe Physical Technical Institute), for stimulating the interest to spectroscopic diagnostics of electron velocity distribution in DEMO, and V.I. Poznyak (NRC "Kurchatov Institute"), for helpful discussion of the EC diagnostics experimental issues.

\section{References}

1. G. Bekefi, Radiation processes in plasmas (Wiley, New York, 1966)

2. M. Bornatici, R. Cano, O. De Barbieri, F. Engelmann, Nuclear Fusion 23, 1153 (1983)

3. P.V. Minashin, A.B. Kukushkin, V.I. Poznyak, EPJ Web of Conferences 32, 01015 (2012)

4. P.V. Minashin, A.B. Kukushkin, V.I. Poznyak, $39^{\text {th }}$ EPS Conference on Plasma Physics 36F, P1.039 (ECA, 2012)

5. I.H. Hutchinson, K. Kato, Nuclear Fusion 26, 179$\underline{191}$ (1986)

6. S. Tamor, Nuclear Technology/Fusion 3, 293 (1983)

7. A.B. Kukushkin, $14^{\text {th }}$ IAEA Conference on Plasma Physics and Controlled Nuclear Fusion Research 2, 35-45 (IAEA, 1992)

8. F. Albajar, M. Bornatici, F. Engelmann, Nuclear Fusion 42, 670-678 (2002)

9. F. Albajar, M. Bornatici, F. Engelmann, Nuclear Fusion 49, 115017 (2009)

10. F. Albajar, M. Bornatici, G. Cortes, J. Dies, et al., Nuclear Fusion 45, 642-648 (2005)
11. A.B. Kukushkin, P.V. Minashin, A.R. Polevoi, Plasma Physics Reports 38, 211-220 (2012)

12. A.B. Kukushkin, P.V. Minashin, A.R. Polevoi, $38^{\text {th }}$ EPS Conference on Plasma Physics 35G, $\underline{\mathrm{P} 4.072}$ (ECA, 2011)

13. A.R. Polevoi, S.Y. Medvedev, T. Casper, Y.V. Gribov, et al., $37^{\text {th }}$ EPS Conference on Plasma Physics 34A, P2.187 (ECA, 2010)

14. K.V. Cherepanov, A.B. Kukushkin, 20 ${ }^{\text {th }}$ IAEA Fusion Energy Conference TH/P6-56 (2004)

15. A.B. Kukushkin, K.V. Cherepanov, L.K. Kuznetsova, E. Westerhof, $14^{\text {th }}$ Joint Workshop on Electron Cyclotron Emission and Electron Cyclotron Heating 374 (Heliotopos Conferences Ltd, 2006)

16. F. Albajar, M. Bornatici, F. Engelmann, $16^{\text {th }}$ Joint Workshop "Electron Cyclotron Emission And Electron Cyclotron Resonance Heating" 215-221 (2010)

17. K.V. Cherepanov, A.B. Kukushkin, $32^{\text {th }}$ EPS Conference on Plasma Physics 29C, P-2.117 (ECA, 2005)

18. P.V. Minashin, A.B. Kukushkin, $40^{\text {th }}$ EPS Conference on Plasma Physics 37D, P4.176 (ECA, 2013)

19. R.W. Harvey, M.G. McCoy, IAEA Technical Committee Meeting on Advances in Simulation and Modeling of Thermonuclear Plasmas 489-526 (IAEA Institute of Physics Publishing, 1992)

20. G. Zhuang, R. Behn, I. Klimanov, P. Nikkola, et al., Plasma Phys. Controlled Fusion 47, 1539 (2005)

21. J. Garcia, G. Giruzzi, J.F. Artaud, V. Basiuk, et al., Nuclear Fusion 48, 075007 (2008)

22. V. Basiuk, J.F. Artaud, F. Imbeaux, X. Litaudon, et al., Nuclear Fusion 43, 822 (2003)

23. A.B. Kukushkin, P.V. Minashin, $36^{\text {th }}$ EPS Conference on Plasma Physics 33E, P-4.136 (ECA, 2009)

24. A.B. Kukushkin, P.V. Minashin, A.R. Polevoi, $23^{\text {rd }}$ IAEA Fusion Energy Conference ITR/P1-34 (2010)

25. F. Albajar, M. Bornatici, F. Engelmann, A.B. Kukushkin, Fusion Science and Technology 55, 76-83 (2009)

26. A.B. Kukushkin, P.V. Minashin, $24^{\text {th }}$ IAEA Fusion Energy Conference TH/P6-25 (2012)

27. G.V. Pereverzev, P.N. Yushmanov, Max-PlanckInstitut für Plasmaphysik Report IPP 5/98 (Garching, 2002)

28. S. Tamor, Science Applications, Inc. Report SAI023-81-189LJ/LAPS-72 (La Jolla, CA, 1981) 